\section{THE DIAGNOSTIC ACCURACY OF THE 2012 SLICC AND THE PROPOSED EULAR/ACR CRITERIA FOR SYSTEMIC LUPUS ERYTHEMATOSUS CLASSIFICATION IS COMPARABLE}

Örjan Dahlström, Christopher Sjöwall*. Linköping University

\subsection{6/lupus-2019-Ism.43}

Background In a joint effort, the European League Against Rheumatism (EULAR) and the American College of Rheumatology (ACR) recently proposed new criteria for the classification of systemic lupus erythematosus (SLE) with the overarching goal to identify potential participants for clinical studies. Herein, we present the first independent evaluation of these criteria in comparison with older classification grounds using an adult Scandinavian study population of confirmed SLE cases and individuals with SLE-mimicking conditions.

Methods We included 56 confirmed SLE cases meeting the 1982 ACR criteria (ACR-82) and/or the Fries "diagnostic principle" (a positive ANA test on at least one occasion plus involvement of at least two defined organ systems) and 55 controls with possible systemic autoimmune disease, including the presence of any SLE-related autoantibody.

Results The proposed EULAR/ACR criteria showed a diagnostic sensitivity of 93\% (95\% confidence interval (CI), 0.830.98) compared with $83 \%(95 \%$ CI, 0.720.91) for the updated ACR criteria from 1997. The diagnostic accuracy of all tested classification grounds was fairly similar, achieving approximately $85 \%$. However, the disease specificity of the EULAR/ ACR criteria reached only 73\% (95\% CI, 0.590.83) which was comparable with the 2012 Systemic Lupus International Collaborating Clinics (SLICC) criteria, $75 \% \quad(95 \% \quad$ CI,

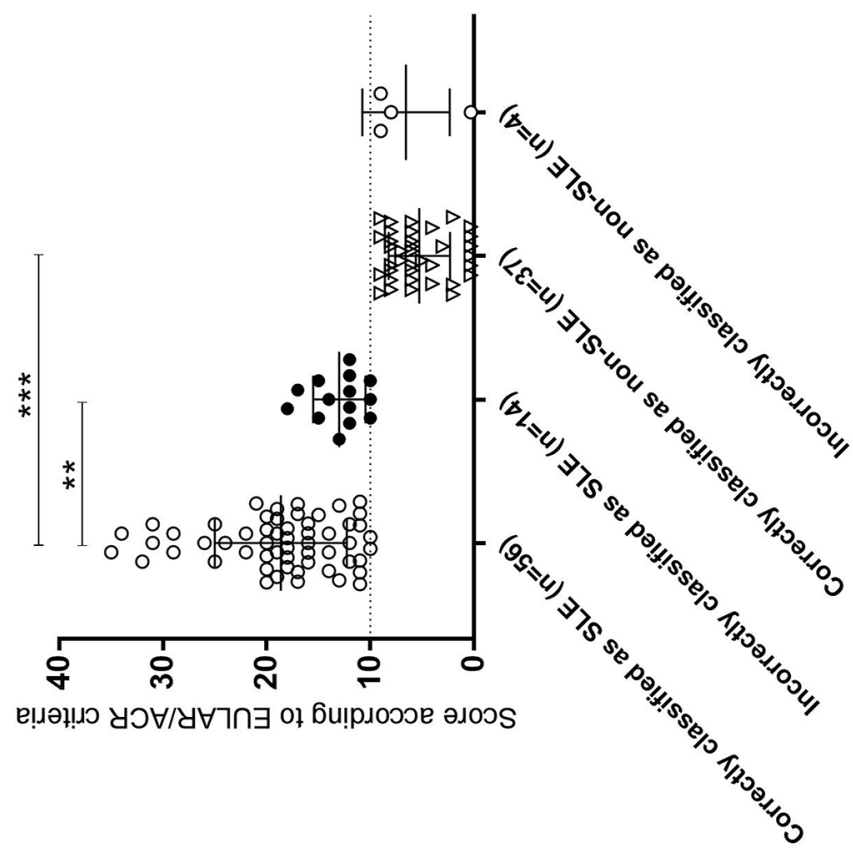

Abstract 43 Figure 1 Weighted scores according to the proposed EULAR/ACR criteria for each of the 111 cases with regard to their classification and final diagnosis. ${ }^{* *} p=0.0005{ }^{* * *} p<0.0001$
0.610.85), but clearly lower than for ACR-82, 94\% (95\% CI, $0.830 .99)$.

Conclusions In this first independent evaluation of a limited number of cases, we found comparable results with respect to diagnostic sensitivity, specificity and accuracy regarding the SLICC-12 and the proposed EULAR/ACR classification criteria. However, their specificity for SLE appeared to be lower compared to ACR-82.

Funding Source(s): This work was supported by grants from the Swedish Rheumatism Association, the County Council of Östergötland, the Swedish Society of Medicine, the King Gustaf Vs 80 year anniversary foundation and the King Gustaf V and Queen Victorias Freemasons foundation.

\section{$44 \quad 47, X X X$ IN LUPUS FAMILIES}

${ }^{1}$ Hal Scofield*, ${ }^{2}$ John B Harley, ${ }^{3}$ Valerie M Harris. ${ }^{1}$ Oklahoma Medical Research Foundation; University of Oklahoma; Oklahoma City US Department of Veterans Affairs Medical Center; ${ }^{2} 4$ Center for Autoimmune Genomics and Etiology and Rheumatology Division, Cincinnati Childrens Hospital Medical Center and the University of Cincinnati; ${ }^{3}$ University of Oklahoam Health Sciences Center

\subsection{6/lupus-2019-Ism.44}

Background Of those with systemic lupus erythematosus (SLE), about $90 \%$ are women. We have reported that the female bias of the disease is related to not phenotypic sex, but instead to the complement of $\mathrm{X}$ chromosomes. Turners women and 46,XY men have low incidence of SLE, while $46, \mathrm{XX}$ women and 47,XXY men have incidences about 10 times higher than individuals with one $\mathrm{X}$ chromosome. In addition, 47, XXX women ( 1 in $\sim 1000$ live female births) are over-represented among SLE patients. We undertook the present study to study the segregation of $47, \mathrm{XXX}$ with SLE within SLE families.

Methods Women with SLE along with their sisters and mothers were enrolled in the Lupus Family Registry and Repository. Subjects were assessed by questionnaire, interview, medical records abstraction and serological examination. All patients met the revised ACR classification criteria, while unaffected family members were shown not to meet these criteria. The number of $\mathrm{X}$ chromosomes was assessed by examination of single nucleotide polymorphism typing of the $\mathrm{X}$ and $\mathrm{Y}$ chromosomes.

Results We studied 2948 women with SLE along with 2116 SLE-unaffected family members, almost entirely sisters and mothers of the SLE patients. Among the total of 5064, we found 7 individuals with 47,XXX, or 1 in 723 (or 0.00138), which was not significantly different from the known 47,XXX population rate $(0.0006$ to 0.0028 (1/1667 to $1 / 357$ by binominal $95 \%$ confidence intervals). All seven 47,XXY subjects had SLE.

Conclusions Among SLE families there was the expected number of women with 47,XXX, approximately 1 in 1000. However, all 47,XXX subjects had SLE. Thus, a 47,XXX karyotype segregated exclusively with SLE within these families. We conclude that carriage of $47, \mathrm{XXX}$ is a powerful risk factor for SLE.

Funding Source(s): Lupus Research Alliance; US Department of Veterans Affairs; National Institutes of Health 